\title{
Reduced Complexity In-phase/Quadrature-phase Turbo Equalisation Using Radial Basis Functions
}

\author{
M. S. Yee, B. L. Yeap and L. Hanzo \\ Dept. of Electr. and Comp. Sc., Univ. of Southampton, SO17 1BJ, UK. \\ Tel: +44-2380-593 125, Fax: +44-2380-593045 \\ Email: lheecs.soton.ac.uk \\ http://www-mobile.ecs.soton.ac.uk
}

\begin{abstract}
A novel reduced complexity Radial Basis Function (RBF) neural network based equaliser, referred to as the In-phase/Quadrature-phase RBF Equaliser (I/Q-RBFEQ), is proposed. The I/Q-RBF-EQ is employed in the context of turbo equalisation (TEQ) assisted by iterative channel estimation. The performance of the I/Q-RBF-TEQ is characterized in a noise limited environment over an equally weighted, symbol-spaced three-path Rayleigh fading channel. The I/Q-RBFTEQ achieved the same performance as the conventional turbo equaliser, while achieving a complexity reduction by a factor of 1.5 and 109.6 for 4-QAM and 16QAM, respectively.
\end{abstract}

\section{Introduction}

The principle of turbo equalisation [3] was contrived by Douillard et al. [3], which was shown to provide performance advantages in the context of a rate $R=0.5$ convolutional coded system, where channel decoding and channel equalisation was performed in unison. More specifically, the turbo equaliser exhibited a performance that was close to that achievable over non-dispersive channels, despite the presence of Inter-Symbol Interference (ISI), when performing the channel equalisation and channel decoding by exchanging information between these two processing blocks. Due to complexity reasons, early turbo equalisation investigations using the conventional trellis-based equaliser (CT-EQ) were constrained to applying Binary Phase Shift Keying (BPSK) and Quadrature Phase Shift Keying (QPSK) modulation schemes [4] and to limited Channel Impulse Response (CIR) durations, since the computational complexity incurred by the CT-EQ is dependent on both the maximum CIR duration and on the modulation mode utilised. Hence, turbo equalisation research has been focused on developing reduced complexity equalisers, such as the low-complexity linear equaliser proposed by Gla- vieux et al. [5], the Radial Basis Function (RBF) equaliser advocated by Yee et al. [12] and the In-phase/Quadraturephase turbo equaliser (I/Q-TEQ) introduced by Yeap [9, 8]. Motivated by these trends, in this contribution we proposed a novel reduced complexity RBF channel equaliser based on the I/Q concept [9] which is referred to as the Inphase/Quadrature-phase RBF Equaliser (I/Q-RBF-EQ) for full response systems.

\section{Principle of $I / Q$ Equalisation}

We denote the modulated signal by $s(t)$, which is transmitted over the dispersive channel characterised by the CIR $h(t)$. The signal is also contaminated by the zero-mean Additive White Gaussian Noise (AWGN) $n(t)$ exhibiting a variance of $\sigma^{2}=N_{o} / 2$, where $N_{o}$ is the single-sided noise power spectral density. The received signal $r(t)$ is then formulated as:

$$
\begin{aligned}
r(t) & =s(t) * h(t)+n(t) \\
& =\left[s_{I}(t)+j s_{Q}(t)\right] *\left[h_{I}(t)+j h_{Q}(t)\right] \\
& +n_{I}(t)+j n_{Q}(t) \\
& =r_{I}(t)+j r_{Q}(t),
\end{aligned}
$$

where

$$
\begin{aligned}
r_{I}(t) & =s_{I}(t) * h_{I}(t)-s_{Q}(t) * h_{Q}(t)+n_{I}(t) \\
r_{Q}(t) & =s_{I}(t) * h_{Q}(t)+s_{Q}(t) * h_{I}(t)+n_{Q}(t),
\end{aligned}
$$

since the CIR $h(t)$ is complex and therefore consists of the I component $h_{I}(t)$ and $\mathrm{Q}$ component $h_{Q}(t)$.

On the same note, $s_{I}(t)$ and $s_{Q}(t)$ are the I and Q components of $s(t)$ in Figure 1, while $n_{I}(t)$ and $n_{Q}(t)$ denote the corresponding AWGN components. Both of the received I/Q signals, namely $r_{I}(t)$ and $r_{Q}(t)$ of Equation 2 become dependent on both $s_{I}(t)$ and $s_{Q}(t)$ due to the crosscoupling effect imposed by the complex channel. Hence a conventional channel equaliser - regardless, whether it is an iterative or non-iterative equaliser - would have to consider the effects of this cross-coupling. 
In this contribution we propose a technique of reducing the complexity of the equaliser by initially neglecting the channel-induced cross-coupling of the received signal's quadrature components and then by compensating for this gross simplification with the aid of the proposed turbo equaliser. This simplification would result in an unacceptable performance degradation in the context of conventional non-iterative channel equalisation, since the turbo iterations allow us to compensate for the above simplification. Then the $I$ and $Q$ components of the decoupled channel output $r^{\prime}(t)$ are only dependent on $s_{I}(t)$ or $s_{Q}(t)$, as portrayed in Figure 2 in the context of the following equations:

$$
\begin{aligned}
r_{I}^{\prime}(t) & =s_{I}(t) * h(t)+n_{I}(t) \\
& =s_{I}(t) * h_{I}(t)+j\left[s_{I}(t) * h_{Q}(t)\right]+n(t) \\
r^{\prime}{ }_{Q}(t) & =-s_{Q}(t) * h(t)+n_{Q}(t) \\
& =-\left(s_{Q}(t) * h_{I}(t)+j\left[s_{Q}(t) * h_{Q}(t)\right]\right)+n(t) .
\end{aligned}
$$

More explicitly, the cross-coupling is facilitated by generating the estimates $\hat{s}_{I}(t)$ and $\hat{s}_{Q}(t)$ of the transmitted signal [5] with the aid of the reliability information generated by the channel decoder and then by cancelling the crosscoupling effects imposed by the channel, yielding $r^{\prime}{ }_{I}(t)$ and $r_{Q}^{\prime}(t)$, respectively, in Figure 2 . In the ideal scenario, where perfect knowledge of both the CIR and that of the transmitted signal is available, it is plausible that the channel-induced cross-coupling between the quadrature components can be removed. However, when unreliable symbol estimates are generated due to the channel-impaired low-confidence reliability values, errors introduced in the decoupling operation. Nonetheless, we will show that the associated imperfect decoupling effects are compensated with the aid of the iterative turbo equalization process and the performance approaches that of the turbo equalizer utilising the conventional trellis-based equalizer, where the crosscoupling is not neglected. As an added benefit, the complexity of the equalisation process is susbtantially reduced. Following the above decoupling operation, the modified complex channel outputs, namely $r^{\prime}{ }_{I}(t)$ and $r_{Q}^{\prime}(t)$, respectively, can be viewed as the result of convolving both quadrature components independently with the complex CIR on each quadrature arm. Consequently, we can equalise $s_{I}(t)$ and $s_{Q}(t)$ independently, hence reducing the number of channel states significantly. Again, note that in Equation 3 we have assumed that perfect signal regeneration and perfect decoupling is achieved at the receiver, in order to highlight the underlying principle of the reduced complexity equaliser.

\section{RBF Assisted Turbo Equalisation}

The RBF network based equaliser is capable of utilizing the a priori information provided by the channel decoder and in turn provide the decoder with the a posteriori information concerning the coded bits [12]. We will now provide

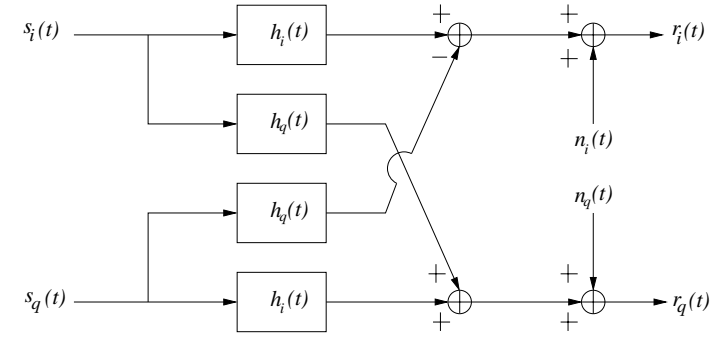

Figure 1: Model of the complex channel. After transmission over the complex channel $h(t)$, the received signal $r(t)$ becomes dependent on the in-phase component $s_{I}(t)$ and quadrature-phase component $s_{Q}(t)$ of the transmitted signal, as expressed in Equations 1 and 2.

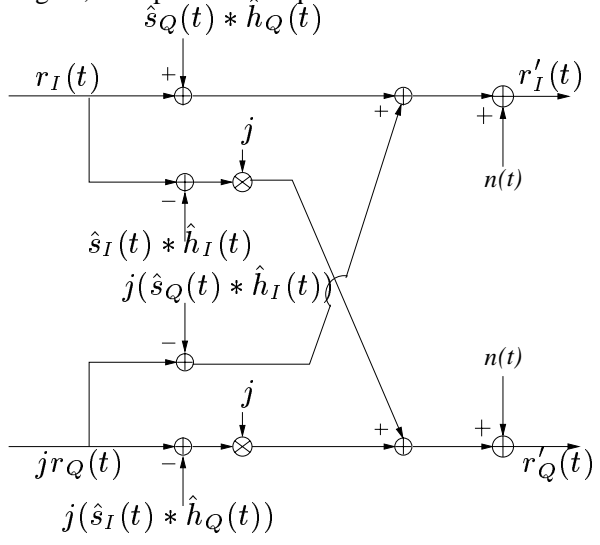

Figure 2: Removing the dependency of $r_{I}(t)$ and $r_{Q}(t)$ on the quadrature components of the transmitted signals, namely $s_{I}(t)$ and $s_{Q}(t)$, to give $r_{I}^{\prime}(t)$ and $r_{Q}^{\prime}(t)$, respectively. In this figure, it is assumed that the CIR estimation is perfect, i.e. $\hat{h}_{I}(t)=h_{I}(t)$ as well as $\hat{h}_{Q}(t)=h_{Q}(t)$ and that the transmitted signals are known, giving $\hat{s}_{I}(t)=s_{I}(t)$ and $\hat{s}_{Q}(t)=s_{Q}(t)$. In this case, perfect decoupling is achieved. However, in practice these estimates have to be generated at the receiver.

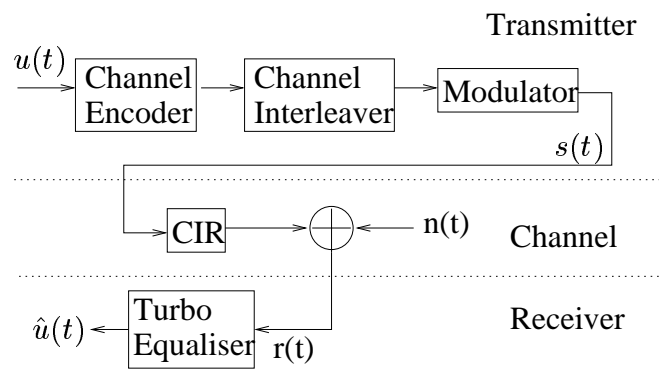

Figure 3: A coded M-QAM system employing a turbo equaliser at the receiver.

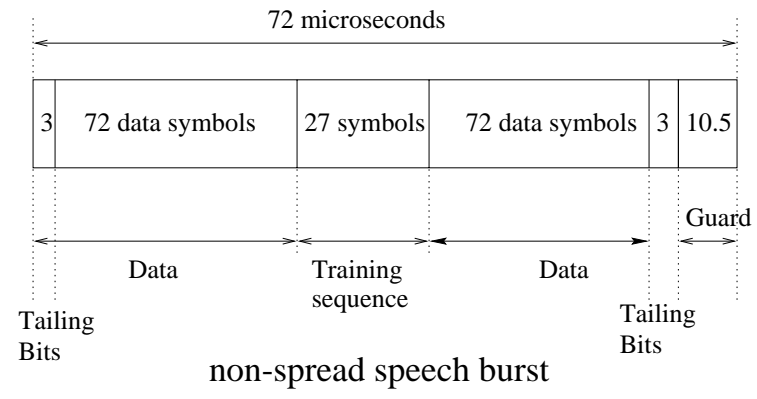

Figure 4: Transmission burst structure of the FMA1 non-spread speech burst of the FRAMES proposal [7]. 
a short description of the RBF based equaliser and how it interacts with the channel decoder.

The conditional probability density function (PDF) of the $i$ th symbol, $i=1, \ldots, M$, associated with the $i$ th subnet of the $M$-ary RBF equaliser having a feedforward order of $m$ is given by [10]:

$$
\begin{aligned}
f_{R B F}^{i}(\mathbf{r}(t))= & \sum_{j=1}^{n_{s}^{i}} w_{j}^{i} \varphi\left(\left|\mathbf{r}(t)-\mathbf{c}_{j}^{i}\right|\right), \\
w_{j}^{i}= & p_{j}^{i}\left(2 \pi \sigma_{\eta}^{2}\right)^{-m / 2}, \\
\varphi(x)= & \exp \left(\frac{-x^{2}}{2 \sigma_{\eta}^{2}}\right) \\
& i=1, \ldots, \mathcal{M}, \quad j=1, \ldots, n_{s}^{i}
\end{aligned}
$$

where $\mathbf{c}_{j}^{i}, \quad \mathbf{w}_{j}^{i}$ and $\varphi(\cdot)$ are the RBF's centers, weights and activation function, respectively, and $\sigma_{\eta}^{2}$ is the noise variance of the channel. The term $\mathbf{r}(t)=\left[\begin{array}{llll}r(t) & r(t-1) & \ldots & r(t-m+1)\end{array}\right]^{T}$ is the $m$-dimensional channel output vector in the memory of the RBF-EQ. The RBF's centers $\mathbf{c}_{j}^{i}$ are assigned to the channel output states $\mathbf{r}_{j}^{i}$. The channel output state, which is the product of the CIR matrix $\mathbf{F}$ and the channel input state $\mathbf{s}_{j}$, is represented as follows [10]: $\mathbf{r}_{j}=\mathbf{H} \mathbf{s}_{j}$, where the z-transform of the CIR $h(t)$ having a memory of $L$ symbols is represented by $H(z)=\sum_{n=0}^{L} h_{n} z^{-n}$ and $\mathbf{H}$ is an $m \times(m+L)$ matrix given by the CIR taps as follows:

$$
\mathbf{H}=\left[\begin{array}{cccccc}
h_{0} & h_{1} & \ldots & h_{L} & \ldots & 0 \\
0 & h_{0} & \ldots & f_{L-1} & \ldots & 0 \\
\vdots & \vdots & & & & \vdots \\
0 & 0 & f_{0} & \ldots & f_{L-1} & f_{L}
\end{array}\right]
$$

The channel input state $\mathbf{s}_{j}$ is given by the $j$ th combination of $(L+m)$ possible transmitted symbols, namely by $\mathbf{s}_{j}=$ $\left[\begin{array}{lllll}s_{j}(t) & \ldots & s_{j}(t-1) & \ldots & s_{j}(t-L-m+1)\end{array}\right]^{T}$. The term $p_{j}^{i}$ in Equation 6 is the probability of occurance of the channel state $\mathbf{r}_{j}^{i}$ and it determines the values of the RBF weights $w_{j}^{i}$. The actual number of channel states $n_{s}^{i}$ is determined by the design of the algorithm that reduces the number of channel states from the optimum number of $\mathcal{M}^{m+L-1}$ [2]. The probability of the channel states $\mathbf{r}_{j}^{i}$ and therefore the weights of the RBF equaliser can be derived from the LLR values of the transmitted bits, as estimated by the channel decoder.

For the I/Q-RBF-EQ, we utilised the principle of I/Q equalisation outlined in Section 2, where two separate RBFEQ is used for the in-phase and quadrature component of the transmitted symbols. The in-phase-RBF-EQ has centers, which consist of the in-phase decoupled channel output $r_{I}^{\prime}(t)$ of Equation 2 and vice-versa for the quadratureRBF-EQ. The number of channel states is reduced, since the decoupled channel output $r^{\prime}(t)$ is dependent on $\sqrt{M}$ possible in-phase or quadrature-phase transmitted symbols instead of the original $M$ symbols. The following section

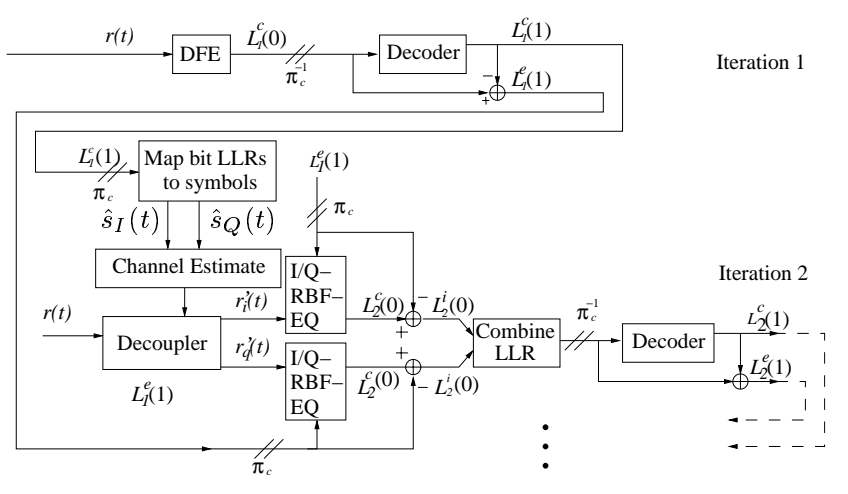

Figure 5: Schematic of the turbo equaliser employing a DFE and a SISO channel decoder in the first turbo equalisation iteration. In subsequent iterations, two I/Q-EQs and one SISO channel decoder are employed. The notation $\pi_{c}$ represents a channel interleaver, while $\pi_{c}^{-1}$ is used to denote a channel deinterleaver.

describes, how the I/Q-RBF-DFE is incorporated into the schematic of the turbo equaliser.

\section{System Overview}

The schematic of the coded $M$-QAM system employing a TEQ at the receiver is shown in Figure 3. The transmitted source bits $u(t)$ are convolutionally encoded, interleaved and mapped to an $M$-QAM symbol. The encoder utilised an $\frac{1}{2}$-rate Recursive Systematic Convolutional (RSC) code having a constraint length of $K=5$ and octal generator polynomials of $G_{0}=35$ and $G_{1}=23$. The transmission burst structure used in this system is the FMA1 non-spread speech burst specified by the Pan-European FRAMES proposal [7], which is shown in Figure 4. In order to decide on the tolerable delay and hence on the depth of the channel interleaver, we considered the maximum affordable delay of a speech system. In our investigations, the transmission delay of the 4-QAM, 16-QAM and 64-QAM systems [6] was limited to approximately $30 \mathrm{~ms}$. This corresponds to 3456 symbols at a symbol rate of 13.9 Kbauds and hence 6912-bit, 13824-bit and 20736-bit random channel interleavers were utilised for 4-QAM, 16-QAM and 64QAM, respectively. A three-path, symbol-spaced fading CIR of equal weights was used, which can be expressed as: $h(t)=0.577+0.577 z^{-1}+0.577 z^{-2}$, where the Rayleigh fading statistics obeyed a normalised Doppler frequency of $3.3615 \times 10^{-5}$. The fading magnitude and phase was kept constant for the duration of a transmission burst, a condition which we refer to as employing transmission burst-invariant fading.

Figure 5 illustrates the schematic of the turbo equaliser utilising two reduced complexity I/Q-RBF-EQs. We express the LLRs of the equaliser and decoder using vector notations, according to the approach of [4], but using different specific notations. The superscript denotes the nature of the LLR, namely ' $c$ ' is used for the composite a posteriori [1] information, ' $i$ ' [1] for the combined channel and extrinsic information and ' $e$ ' [1] for the extrinsic information. 


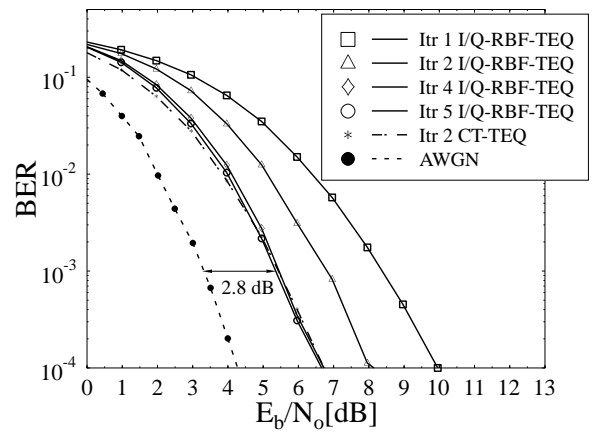

Figure 6: Performance of the I/Q-RBF-TEQ using iterative CIR estimation and the CT-TEQ having perfect channel estimation for a convolutional-coded 4QAM system possessing a channel interleaving depth of 69124 bits over the equally-weighted three-path Rayleigh fading CIR using a normalised Doppler frequency of $3.3 \times 10^{-5}$. The initial LMS CIR estimation step-size is 0.1 and the subsequent step-size is 0.01 .

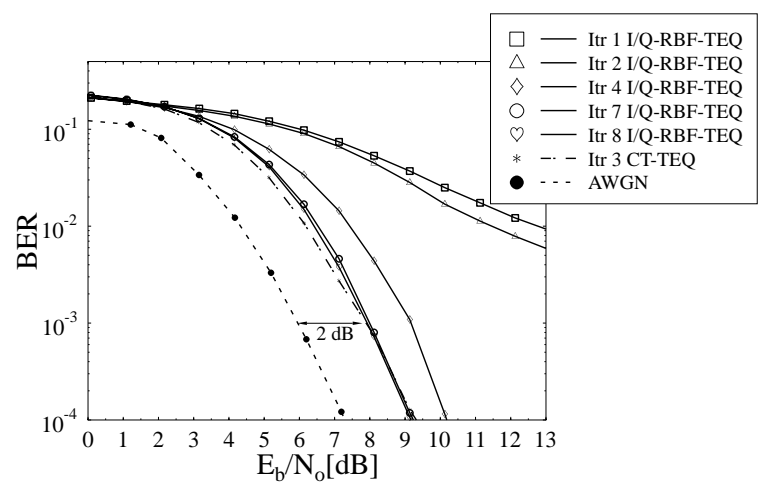

Figure 7: Performance of the I/Q-RBF-TEQ using iterative CIR estimation and the CT-TEQ having perfect channel estimation for a convolutional-coded 16QAM system possessing a channel interleaving depth of 13824 bits over the equally-weighted three-path Rayleigh fading CIR using a normalised Doppler frequency of $3.3 \times 10^{-5}$. The initial LMS CIR estimation step-size is 0.05 and the subsequent step-size is 0.01 .

The subscripts in Figure 5 are used to represent the iteration index, while the argument within the brackets ( ) indicates the index of the receiver stage, where the equalisers are denoted as stage 0 , while the channel decoder as stage 1 .

The conventional Decision Feedback Equaliser (DFE), as seen in Figure 5 is used for the first turbo equalisation iteration for providing soft decisions in the form of the LLR $L_{1}^{c}(0)$ to the channel decoder. Invoking the DFE at the first iteration constitutes a low-complexity approach to providing an initial estimate of the transmittd symbols, as compared to the more complex I/Q-RBF-TEQ. The SoftIn/Soft-Out (SISO) channel decoder of Figure 5 generates the a posteriori LLR $L_{1}^{c}(1)$ and from that, the extrinsic information of the encoded bits $L_{1}^{e}(1)$ is extracted. In the next iteration, the a posteriori LLR $L_{1}^{c}(1)$ is used for regenerating estimates of the I and Q components of the transmitted signal, namely $\hat{s}_{I}(t)$ and $\hat{s}_{Q}(t)$, as seen in the 'MAP bit LLRs to symbols' block of Figure 5. The a posteriori information was transformed from the log domain to modulated symbols using the approach employed in [5]. The estimated

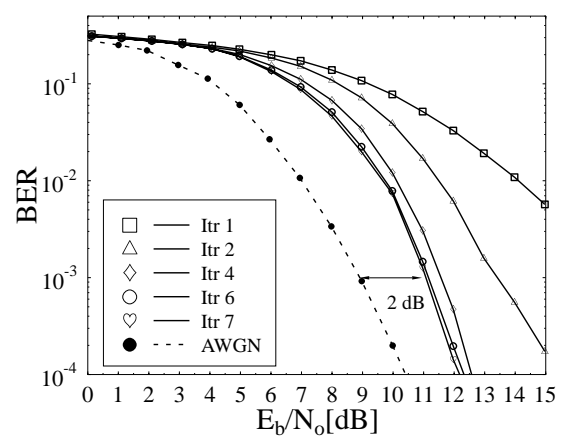

Figure 8: Performance of the I/Q-RBF-TEQ using perfect CIR estimation for a convolutional-coded 64QAM system possessing a channel interleaving depth of 20736 bits over the equally-weighted three-path Rayliegh fading CIR using a normalised Doppler frequency of $3.3 \times 10^{-5}$.

transmitted quadrature components $\hat{s}_{I}(t)$ and $\hat{s}_{Q}(t)$ are then convolved with the estimate of the CIR $h(t)$. At the decoupler block of Figure 5, the resultant signal is used for removing the cross-coupling effect - seen in Equation 2 according to Equation 3 from both quadrature components of the transmitted signal, yielding $r^{\prime}{ }_{I}(t)$ and $r_{Q}^{\prime}(t)$.

After the decoupling operation, $r^{\prime}{ }_{I}(t)$ and $r_{Q}^{\prime}(t)$ are passed to the I/Q-RBF-EQ in the schematic of Figure 5. In addition to these received quadrature signals, the I/QRBF-EQ also processes the a priori information received — which is constituted by the extrinsic LLRs $L_{1}^{e}(1)$ derived from the previous iteration - and generates the a posteriori information $L_{2}^{c}(0)$. Subsequently, the combined channel and extrinsic information $L_{2}^{i}(0)$ is extracted from both I/QRBF-EQs in Figure 5 and combined, before being passed to the Log-MAP channel decoder. As in the first turbo equalisation iteration, the a posteriori and extrinsic information of the encoded bits, namely $L_{2}^{c}(1)$ and $L_{2}^{e}(1)$, respectively, are evaluated. Subsequent turbo equalisation iterations obey the same sequence of operations, until the iteration termination criterion is met.

\section{Results and Discussion}

In our simulations, the Jacobian RBF DFE of [11] was employed, which reduced the complexity of the RBF-EQ by utilising Jacobian logarithmic function and decision feedback for RBF-center selection [10]. Note that the I/Q-EQ scheme reduces the effect of error propagation, since the set of centers to be selected using the DFE mechanism is reduced from $M^{n}$ to $M^{n / 2}$ [10]. The feedforward and feedback order of the RBF DFE was three and two, respectively, while that of the conventional DFE was fifteen and four. We employed iterative LMS-based CIR estimation for both 4QAM and 16QAM in Figure 6 and 7, respectively. An initial step-size of 0.1 and 0.05 was set for 4QAM and 16QAM, respectively, in order to provide a rough estimate of the CIR. This initial CIR estimate was utilised by the conventional DFE employed during the first turbo equalisation. In the subsequent iterations, the CIR was re-estimated 


\begin{tabular}{|cll|}
\hline & Log-MAP & Jacobian RBF \\
\hline sub. and add. & $n_{s, f}(6 \mathcal{M}+2)-3$ & $n_{s, f}+$ \\
& & $\mathcal{M} n_{s}^{i}(m+2)-4$ \\
mult. and div. & $2 n_{s, f}$ & $n_{s, f}$ \\
\hline
\end{tabular}

Table 1: Computational complexity of generating the a posteriori LLR for the Log-MAP equaliser and the Jacobian RBF equaliser [11]. The RBF equaliser order is denoted by $m$ and the number of RBF nodes is $n_{s}^{i}$. The notation $n_{s, f}=\mathcal{M}^{L+1}$ indicates the number of trellis transition for the Log-MAP equaliser and also the number of scalar channel states for the Jacobian RBF equaliser.

and refined using a smaller step-size of 0.01 . Figure 6 and 7 also present the performance of the CT-TEQ in conjunction with perfect CIR information. We defined the critical number of turbo equalisation iterations, as the number, where no further significant performance improvement can be obtained upon invoking further iterations. We found that both the CT-TEQ and the I/Q-RBF-TEQ provided a similar performance at the corresponding critical number of iterations. The performance of the I/Q-RBF-TEQ at the critical number of iteration using iterative CIR estimation at BER $=10^{-3}$ was about $2.8 \mathrm{~dB}$ and $2 \mathrm{~dB}$ from the decoding performance curve recorded over the non-dispersive Gaussian channel, as shown for 4QAM and 16QAM in Figures 6 and 7 , respectively. Following the system complexity study of [12] and considering the number of critical iterations needed, the complexity of the I/Q-RBF-TEQ imposed by the equaliser and decoder components was found to be a factor of 1.5 and 109.6 lower, than that of the CT-TEQ for 4-QAM and 16-QAM, respectively, based on the general complexity expressions of Table 1 .

The performance of our 64-QAM system for transmission over the same Rayleigh fading channel but in conjunction with perfect channel estimation shows in Figure 8 that it is only $2 \mathrm{~dB}$ away from the decoding performance curve recorded for seven iterations over the non-dispersive Gaussian channel at $\mathrm{BER}=10^{-3}$. We could not provide the performance curve of the CT-TEQ, since this scheme was excessively complex. Assuming that the critical number of iteration for the 64QAM CT-TEQ is two, the I/Q-RBF-TEQ provides a complexity reduction by a factor of 3313 .

The performance of the I/Q-RBF-EQ and that of the trellis-based I/Q-TEQ of [9] was found to be similar, although I/Q-RBF-EQ provided a complexity reduction factor of 1.2, 2.2 and 7.8 for 4QAM, 16QAM and 64QAM respectively.

\section{Conclusion}

Our simulation results show significant complexity reductions for the I/Q-RBF-EQ when compared to the conventional CT-EQ, while achieving virtually the same performance. This is demonstrated in Figure 6 and 7, where the complexity reduction factor was 1.5 and 109.6 for 4QAM and 16QAM, respectively. The achievable complexity reduction increases for higher-dispersion channels and for high-order modulation schemes.

\section{References}

[1] L. R. Bahl, J. Cocke, F. Jelinek, and J. Raviv: Optimal decoding of linear codes for minimising symbol error rate; IEEE Transactions on Information Theory, 20:284287, March 1974.

[2] S. Chen, B. Mulgrew, and S. McLaughlin: Adaptive Bayesian equalizer with decision feedback; IEEE Transactions on Signal Processing, 41(9):2918-2927, September 1993.

[3] C. Douillard, M. Jézéquel, and C. Berrou: Iterative correction of intersymbol interference: Turbo-equalization; $\mathrm{Eu}$ ropean Transactions on Telecommunication, 6(5):507-511, September/October 1995.

[4] M. J. Gertsman and J. L. Lodge: Symbol-by-symbol MAP demodulation of CPM and PSK signals on Rayleigh flatfading channels; IEEE Transactions on Communications, 45:788-799, July 1997.

[5] A. Glavieux, C. Laot, and J. Labat: Turbo equalization over a frequency selective channel; In International Symposium on Turbo Codes, pages 96-102, Brest, France, 1997.

[6] L. Hanzo, W. Webb, and T. Keller: Single- and Multi-carrier Quadrature Amplitude Modulation: Principles and Applications for Personal Communications, WLANs and Broadcasting; John Wiley and Sons, Ltd., 2000.

[7] A. Klein, R. Pirhonen, J. Sköld, and R. Suoranta: FRAMES multiple access mode 1 - wideband TDMA with and without spreading; In Proceedings of PIMRC'97, pages 37-41, September 1997.

[8] B. L. Yeap: Turbo Equalisation Algorithm for Full and Partial Response Modulation; $\mathrm{PhD}$ thesis, University of Southampton, January 2000.

[9] B. L. Yeap, C. H. Wong, and L. Hanzo: Reduced complexity in-phase/quadrature-phase turbo equalisation; In IEEE International Communications Conference, Helsinki, Finland, 11 - 14 June 2001.

[10] M. S. Yee and L. Hanzo: Multi-level radial basis function network based equalisers for Rayleigh channels; In Proceedings of IEEE Vehicular Technology Conference, pages 707-711, Houston, USA, 16-19 May 1999. IEEE.

[11] M. S. Yee, T. H. Liew, and L. Hanzo: Block turbo coded burst-by-burst adaptive radial basis function decision feedback equaliser assisted modems; In Proceedings of IEEE Vehicular Technology Conference, volume 3, pages 16001604, Amsterdam, Netherlands, September 1999.

[12] M. S. Yee, B. L. Yeap, and L. Hanzo: Radial basis function assisted turbo equalisation; In Proceedings of IEEE Vehicular Technology Conference, pages 640-644, Japan, Tokyo, 15-18 May 2000. IEEE. 